\title{
Creatinine Increase Caused by Etamsylate Interference: A Case Report of Clinical Pharmaceutical Care
}

\author{
Lejia Xu ${ }^{1}$, Xingling Liu ${ }^{1}$, Danwei Zheng ${ }^{1}$, Jiawen Huang ${ }^{2}$, * \\ ${ }^{1}$ Department of Pharmacy, The Third Affiliated Hospital of Sun Yat-sen University, Guangzhou, China \\ ${ }^{2}$ Department of Pharmacy, The First Affiliated Hospital, Jinan University, Guangzhou, China
}

Email address:

eudaemoniay@139.com (Jiawen Huang)

${ }^{*}$ Corresponding author

\section{To cite this article:}

Lejia Xu, Xingling Liu, Danwei Zheng, Jiawen Huang. Creatinine Increase Caused by Etamsylate Interference: A Case Report of Clinical Pharmaceutical Care. Science Journal of Clinical Medicine. Vol. 10, No. 2, 2021, pp. 38-41. doi: 10.11648/j.sjcm.20211002.14

Received: May 13, 2021; Accepted: May 28, 2021; Published: June 3, 2021

\begin{abstract}
Background: The accuracy of test results is closely related to the clinical diagnosis and treatment, while drugs may interfere with the test results. When the abnormal value of results occured, it is important to analyze the drug-related interferential factors. Objective: To remind clinicians and pharmacists to eliminate interference factors when finding abnormal results by recording the cause analysis process of a patient with abnormally elevated creatinine. Methods: In this case of an abnormally elevated creatinine in a patient treated with vancomycin, the clinical pharmacist analyzed the reasons of patients' creatinine elevation, especially vancomycin-associated kidney injury and other interfering factors of combination drugs in the tests. Results: The clinical pharmacist Clinical pharmacists found that acute kidney damage was not caused by vancomycin, the "real killer" was the etamsylate interference factors in laboratory test of creatinine value. This discovery enabled the patient's antibiotic treatment to proceed smoothly. Conclusions: As a clinical pharmacist, it is necessary to understand the medication situation of patients and be familiar with the common types of "drug interference detection". With these advantages in drug knowledge, clinical pharmacists can assist clinicians to identification and judgment of adverse drug reactions, and play an important role in the treatment of patients.
\end{abstract}

Keywords: Vancomycin, Creatinine, Etamsylate, Clinical Pharmacist

\section{Introduction}

With the development of medical science, more and more drugs and testing items are applied in clinical practice to provide scientific and professional basis for the diagnosis and treatment of patients. Therefore, the accuracy of test results is closely related to the correctness of clinical diagnosis and treatment. However, drugs, including main drug ingredients or excipients in the preparation, may interfere with the test results, such as false positive or false negative, false normal value or false outlier value, high value or low value, or a variety of unexplained results. Studies as far back as 1962 suggested that around $7 \%$ of tests were disrupted when patients took one drug, $16.7 \%$ when they took two drugs and $66.7 \%$ when they took three or four drugs [1]. According to statistics, up to 15000 kinds of drugs have interference effect on the test. For example, most antibiotics can cause the increase of leukocytes, neutrophils, thrombocytopenia and eosinophils [2]. Vitamin $\mathrm{C}$, can reduce blood glucose measurements by $65 \%$ at a blood concentration of $450 \mathrm{mg} / \mathrm{L}$ [3]. Estrogen drugs can reduce the experimental value of glucose tolerance. From the case we reported below, it can be seen that clinical pharmacists need to give full play to their advantages in drug knowledge and assist the clinic to eliminate drug-related factors when abnormal test results occur, which is of great significance to assist clinical diagnosis and treatment, avoid missed diagnosis and misdiagnosis, and improve the level of rational drug use.

\section{Case Presentation}

A 74-year-old man was referred to our department due to "recurrent cough and sputum accompanied by shortness of breath for 2 years and exacerbation accompanied by fever for 2 weeks". The patient began to develop cough and sputum 
with no obvious inducement 2 years ago, which was intermittent dry cough, mainly at night, with white mucus and sputum. The patient also had shortness of breath, and could only climb 2 flights of stairs. Two weeks before admission, patients began to develop fever with no obvious inducement, mostly in the afternoon, with the highest temperature of $38.5^{\circ} \mathrm{C}$. The results of chest $\mathrm{CT}$ examination in the local hospital showed chronic bronchitis and emphysema in both lungs. Extensive interstitial fibrous changes in the right middle and upper lung and dorsal segment of the lower lobe were accompanied by bronchiectasis and secondary infection. Mediastinal lymph node enlargement; Right pleural thickening and adhesion. The patients were given piperacillin tazobactam, moxifloxacin, imipenem, vancomycin and other symptomatic supportive treatment against infection, with poor effect, and recurrent fever was still present.

On admission, upper limb blood pressure was 156/86 $\mathrm{mmHg}$, heart rate was 94 beats/min, respiratory rate was 23 breaths $/ \mathrm{min}$, the temperature was $36.5^{\circ} \mathrm{C}$. Bilateral breathing sounds are thick, and rales can be heard on both sides, especially on the right side. Initial laboratory studies showed $20.37 \times 10^{\wedge} 9$ leukocytes/L, a hemoglobin of $107.2 \mathrm{~g} / \mathrm{L}$, platelets of $243.6 \times 10^{\wedge} 9 / \mathrm{L}$, C-reaction protein (CRP) of $99.5 \mathrm{mg} / \mathrm{L}$, procalcitonin(PCT) of $0.52 \mathrm{ng} / \mathrm{mL}$, serum creatinine of $60 \mu \mathrm{mol} / \mathrm{L}$, and normal liver enzymes. The admission diagnosis was respiratory failure (type I).

After admission, the patient was given non-invasive ventilator assisted ventilation. After $3 g * q 8 h$ of cefoperazone and sulbactam injection combined with $0.6 \mathrm{~g} * \mathrm{qd}$ of levofloxacin injection, the symptoms were still not significantly improved, and the fever was still repeated, with a fever peak of $38.7^{\circ} \mathrm{C}$.

Day 4 after admission, the patient presented bright red blood sputum with a small amount of about $10 \mathrm{ml}$. The sputum culture indicated the growth of Aspergillus. Blood culture showed gram-positive cocci. Considering that the patient's infection was not under control and his condition was serious, the anti-infection regimen was changed to vancomycin injection $1 \mathrm{~g} * \mathrm{q} 12 \mathrm{~h}+$ meropenem injection $1 \mathrm{~g} * \mathrm{q} 8 \mathrm{~h}+$ caspofungine injection 50mg*qd. The patient's symptoms improved and the fever peak decreased, but hemoptysis was still present.

The creatinine level increased to $124.7 \mu \mathrm{mol} / \mathrm{L}$ on Day 8 , and the physician considered the possibility of vancomycin-related acute renal impairment. After the clinical pharmacist looked up the medication history in detail, it was found that the patient had hemoptysis early that day and was given normal saline $500 \mathrm{ml}+$ aminomethylenic acid 300mg + phenolic sulfoethylamine $2 \mathrm{~g}$ intravenous drops for treatment at 4:15AM. The infusion time lasted for longer than 3 hours nevertheless the test blood drawing time was around 6:00, suggesting that the creatinine elevation may have been due to the interference of phenol-sulfoethylamine with the test. The doctor accepted the suggestion and reexamined the creatinine without stop the Vancomycin administration. The result confirmed a normal level of creatinine of $58 \mu \mathrm{mol} / \mathrm{L}$. Therapeutic Drug Monitoring (TDM) showed a valley concentration of vancomycin of $10 \mathrm{mg} / \mathrm{L}$ the next day. After three weeks of continuation of the anti-infective treatment, the patient's symptoms improved and were transferred to a local hospital for further treatment.

\section{Discussion}

Creatinine in human body includes exogenous creatinine from food intake and endogenous creatinine produced by muscle tissue metabolism, which is the final metabolite of creatine and phosphocreatine in muscle. Normally, the production of creatinine is constant and almost all of it is excreted through the kidneys. The level of serum creatinine mainly depends on the glomerular filtration capacity, that is, the amount of creatinine excreted by the kidneys. Therefore, the increase of serum creatinine indicates that the patient's renal function may be impaired, and serum creatinine is an important indicator of renal function [4]. There are a number of factors that contribute to elevated serum creatinine, including kidney disease, high blood pressure, diabetes, trauma, drug-induced kidney damage and so on, and measuring interference should never been ignore. The patient had normal creatinine at admission $(60 \mu \mathrm{mol} / \mathrm{L})$ and no previous history of renal disease, which precluded the presence of chronic renal insufficiency. The serum creatinine elevation was sudden. After admission, vancomycin treatment was started on Day4 with a dose of $1 \mathrm{~g} * \mathrm{q} 12 \mathrm{~h}$. Biochemistry indicated that creatinine increased to $124.7 \mu \mathrm{mol} / \mathrm{L}$ on Day 8 . The drugs involved in the treatment regimen included vancomycin, meropenem, caspofungin, ambroxol, acetylcysteine, etamsylate and aminomethylbenzoic acid, vancomycin seemed to be the most prone to renal toxicity. In addition, the elevation of creatinine in this patient was in time sequence with the application of vancomycin, so it was necessary to first investigate whether it was vancomycin-related nephrotoxicity.

The incidence of acute renal insufficiency caused by vancomycin is about $0.5 \%$. Vancomycin-associated nephrotoxicity was considered when the serum creatinine increased $0.5 \mathrm{mg} / \mathrm{L}$ or increased by more than $50 \%$ of baseline from the pre-treatment baseline for two consecutive periods of treatment with vancomycin, or when the creatinine clearance rate decreased by more than $50 \%$ from baseline for two consecutive periods, excluding other pathological factors[5]. Risk factors for vancomycin nephrotoxicity include high dose administration, combination with other renal damage agents, basic renal function, and the duration of vancomycin [6]. Among them, the serum valley concentration of vancomycin $>15 \mu \mathrm{g} / \mathrm{mL}$ was an independent risk factor for acute renal damage [7]. The relationship between the duration of vancomycin and acute kidney injury is still controversial. Some studies believe that there is no significant correlation between the two [8], but some studies have shown that the duration of treatment $\geq 7$ or 14 days may increase the incidence of acute kidney injury [9]. It is generally believed that acute renal damage caused by vancomycin is most likely to occur at about 5-7 days after administration [10], and oliguria and other symptoms may occur before creatinine increase. The 
combination of other nephrotoxic drugs, such as aminoglycosides, diuretics, ACEI/ARB or immunosuppressive agents, may increase the incidence of acute kidney injury [11]. In addition, the pathophysiological status of patients, such as low body weight, low basic renal function, and advanced age, may increase the risk of vancomycin-related nephrotoxicity [12]. The patient in this case had no risk factors for vancomycin-related nephrotoxicity, no symptoms such as oliguria, and no proportionately elevated urea nitrogen. There was insufficient evidence to judge vancomycin-induced nephrotoxicity solely due to increased creatinine. The subsequent valley concentration of vancomycin was $10 \mathrm{mg} / \mathrm{L}$, which also confirmed that the concentration was in proper range.

The creatinine determination method adopted in our hospital is the piric acid method, which is affected by many factors, including $\alpha$-ketoglutaric acid, bilirubin, hemoglobin, dopamine, vitamin $\mathrm{C}$ and so on [13]. The determination of creatinine by picric acid method is due to the fact that creatinine exists in the form of enol creatinine in alkaline medium, which has a strong reductivity. It reacts with alkaline picric acid to produce an orange picric creatinine complex. Phenolic sulfoethylamine, like vitamin $\mathrm{C}$, reacts directly with picric acid as pseudocreatinine to produce orange color, resulting in positive interference [14]. Studies have shown that when the concentration of phenol-sulfoethylamine is above $0.07 \mathrm{~g} / \mathrm{L}$, it will interfere the determination of creatinine by picric acid method. When the concentration is $0.3 \mathrm{~g} / \mathrm{L}$ (the highest blood concentration of $1.5 \mathrm{~g}$ phenol-sulfoethylamine administration), the creatinine result can be increased by $18.5 \%$ [15]. According to the drug instructions, after $1 \mathrm{~h}$ of phenol-sulfoethylamine injection, the blood concentration reaches the peak, the protein binding rate reaches $90 \%$, the action time lasts $4-6 \mathrm{~h}, 80 \%$ is excreted from the kidney as a type, a small part is excreted from bile and feces, and the half-life of intravenous infusion is $1.9 \mathrm{~h}$ [16]. As a result, the patient needed three half-life periods (approximately 6 hours) of clearance after $2 \mathrm{~g}$ administration to get the plasma concentration below $0.07 \mathrm{~g} / \mathrm{L}$ to avoid interference with the creatinine measurement. The clinical pharmacist noted that phenol-sulfoethylamine was still in the process of infusion when the blood samples with abnormal creatinine values were collected, so it was likely that phenol-sulfoethylamine interfered with the determination. The creatinine was later confirmed as $58 \mu \mathrm{mol} / \mathrm{L}$, so the renal function damage caused by vancomycin was ruled out, and the anti-infection therapy of vancomycin was continued.

\section{Conclusion}

The accuracy of test results is closely related to the accuracy of clinical diagnosis and treatment. Correct and objective laboratory test results are of great significance in guiding clinical diagnosis and treatment, avoiding missed diagnosis and misdiagnosis, and reducing patients' medical expenses. Drugs interference refers to the result of the test itself and its metabolites cause human physiological, biochemical and pathological aspects of complex changes which might affect the clinical test results and application test reagent, expression is the result of false positives or false negatives, false normal or false outliers, high value or partial low, or a variety of cannot explain the results. As a clinical pharmacist, it is necessary to understand the medication situation of patients and be familiar with the common types of "drug interference detection". The interference of many drugs to test results is often positively correlated with the blood drug concentration, so it is necessary to be familiar with the blood drug concentration level, half-life, excretion route and clearance rate of drugs, so that the test sampling should avoid the peak of blood drug as far as possible. Clinical pharmacists, with their advantages in drug knowledge, can assist clinicians in the identification and judgment of adverse drug reactions, and play an important role in the treatment of patients.

\section{References}

[1] Yong He, Zhiguang Tu. Drug interference with the results of laboratory tests $[\mathrm{J}]$. International Journal of Laboratory Medicine, 2003, 24 (2): 122-123.

[2] Daiyan Kong, Xiaoli Dai, Zheng Zhang. Adverse reactions of cephalosporins $[\mathrm{J}]$. Chinese Journal of Hospital Pharmacy, 2001, 21 (8): 511.

[3] Suzhong Dai. Interference of drugs to biochemical test results [J]. Journal of Practical Medical Techniques, 2005, 12 (2): 392-393.

[4] Qianyu Liu. Clinical progress of serum creatinine concentration [J]. International Journal of Laboratory Medicine, 1994, 15 (6): 255-258.

[5] Rybak M, Lomaestro B, Rotschafer JC, et al. Therapeutic monitoring of vancomycin in adult patients: a consensus review of the American Society of Health-System Pharmacists, the Infectious Disease Society of America, and the Society of Infectious Diseases Pharmacists. [J] Am J Health Syst Pharm, 2009, 66 (1): 82-89.

[6] Xiaoxiao Sun, Hua Shao, Youqun Wang. Research progress of vancomycin nephrotoxicity [J]. Chinese Journal of Clinical Pharmacology and Therapeutics, 2014, 19 (5): 597-600.

[7] Wei Li, Na Yin, Hao Jiang, et al. Analysis of drug use and pharmaceutical care for an elderly patient with plasma exchange for vancomycin-induced acute kidney injury $[\mathrm{J}]$. China Pharmacy, 2018, 29 (19): 2704-2708.

[8] Meaney CJ, Hynicka LM, Tsoukleris MG. Vancomycin-associated nephrotoxicity in adult medicine patients: incidence, outcomes, and risk factors $[\mathrm{J}]$. Pharmacotherapy, 2014, 34 (7): 653-661

[9] Wunderink RG, Niederman MS, Kollef MH, et al. Linezolid in methicillin-resistant Staphylococcusaureus nosocomial pneumonia: a randomized, controlled study [J]. Clin Infect Dis, 2012, 54 (5): 621-629.

[10] Jeffres MN, Isakow W, Doherty JA, et al. A retrospective analysis of possible renal toxicity associated with vancomycin in patients with health care-associated methicillin-resistant Staphylococcus aureus pneumonia [J]. Clin Ther, 2007, 29 (6): 1107-1115. 
[11] Ting Mao, Jiying Li, Shenghong Wang, et al. Systematic evaluation of risk factors for intravenous dripping of vancomycin-induced acute kidney injury in Chinese patients [J]. China Pharmacy, 2018, 29 (13): 1836-1840.

[12] Nian Ye. Clinical pharmacists participated in the pharmaceutical care of vancomycin-related acute kidney injury [J]. Journal of Military Surgeon in Southwest China, 2019, 21 (06): 558-560, 545 .

[13] Lingbo Shi, Longshun Lin. The interference of common methods in determination creatinine $[\mathrm{J}]$. Chinese Journal of Laboratory Medicine, 2001, 24 (2): 102-104.
[14] Xinmin Gu. The influence factors of creatinine determine with alkaline picric acid method [J]. Journal of traditional Chinese and western medicine, 2005, 3 (4): 356.

[15] Yongjiang Cheng, Li Li, Haiming Chen. The interference of different methods on testing serum creatinine by ethamsylate [J]. Laboratory Medicine and Clinic, 2011, 8 (8): 1939-1941.

[16] Drug instruction of etamsylate injection, Tianjin Jinyao Pharmaceutical Co. Ltd. 